\title{
A Multichain Slip-Spring Model with Fluctuating Number of Entanglements Based On Single-Chain Slip-Spring Model
}

\author{
Teng MA ${ }^{1}$, Hui-Feng $\mathrm{TAN}^{1,2}$ and Lin $\mathrm{YU}^{1}$ \\ 1 Centre for Composite Materials and Structures, Harbin Institute of Technology, Harbin \\ 150080,China

\begin{abstract}
2National Key Laboratory of Science and Technology on Advanced Composites in Special Environments, Harbin Institute of Technology, Harbin 150080, China
\end{abstract}

Keywords: Single-chain slip-spring model, Entanglement, Excluded volume interaction.

\begin{abstract}
We present a multichain slip-spring model with fluctuating number of entanglements for dynamics of dense polymer melts, which is based on the single chain slip-spring model by Likhtman. By introducing the exclude volume interactions, a multichain version of slip-spring model can be presented with little modification. With this extension, inhomogeneous polymer system can be described properly. The renewal rules for the slip-spring satisfy the detailed balance condition and the number of slip-springs is no longer constant but controlled through a chemical potential. This completion can predict the Possion distribution of $\mathrm{Z}$ under equilibrium states and the decrease in the number of entanglements when applied a steady shear flow. The results agree with theoretical predictions and experimental phenomenon. The model bridges the single chain slipspring model and multichain slip-spring model.
\end{abstract}

\section{Introduction}

Understanding of the dynamics of entangled polymer is very important for many industrial applications. However, for the wide range of spatial and temporal scales involved in the characterization of entangled polymer, theoretical modelling of such material is a difficult challenge. A most successful and advanced models was the tube model of Doi and Edwards[1-4], in which a probe Rouse chain is restricted in a tube formed by the topological constraints imposed by surrounding chains. The chain diffusion along the backbone more easily than lateral to the backbone, which is known as reptation. Based on this reptation idea, many important additional physical ideas, such as constraint release(CR)[5], contour-length fluctuations(CLF)[6], convective constraint release(CCR)[7], have been incorporated into tube model. These improvements have enriched the tube theory to account for many additional relaxation mechanisms. Tube model reveals the dominant relaxation mechanism and could describe numerous characteristic phenomena of entangled polymers successfully. However, it still failed in many respects, such as incorrect prediction for steady shear flow and it's difficult to be extended to various chain architectures. Tube model met these troubles mainly because it employ a single-chain motion to express multichain dynamics, thus introducing excessive assumptions and approximations.

For the shortness of tube model, many researchers have transferred to slip-link model, which incorporate constraint release and fluctuations in the tube length in a more natural way. There are several excellent implementations of slip link models in the literature, such as the primitive chain network (PCN) model of Masubuchi and co-workers[8, 9], the TIEPOS approach of RamírezHernández and co-workers[10, 11], the DSM of Schieber and co-workers[12-14]. Likhtman[15] developed a dynamic one-chain slip-spring model of entangled polymers suitable for Brownian dynamics simulations. This model can describe the results from NSE, linear rheology and diffusion experiments properly. Many models extend Likhtman's model for its flexibility, extensibility and implementation simplicity. To simulate large scale or long time macroscopic phenomena, Uneyama slightly modified Likhtman's model to be suitable for simulations on a GPU[16]. The total number of slip-springs could fluctuates and controlled by a pseudo-chemical potential, which is originally introduced by Schieber [17]. However, the model still is single-chain model that it's hard to use it to 
simulate filled entangled system. Biondo et.al investigated the nonlinear rheological properties of Likhtman's model and extend the model to study inhomogeneous system[18]. The extension is flexible and simply implemented by introducing excluded volume interactions in a mean field. However, the total number of the slip-springs in their model is constant, which seems not true during shear flow. In this work, we will use Biondo's method to achieve a multichain model from Likhtman's model. Meanwhile the fluctuation in the number of slip-springs should be accomplished with Uneyama's method.

It is important to highlight that Ramírez-Hernández et al. developed a version slip-spring model refer to as "Theoretically informed entangled polymer simulations" (TIEPOS), which can also describe the dynamics of inhomogeneous systems with fluctuating number of entanglements well[19]. Our work stress the extension from single-chain slip-spring model directly, thus the anchors of each chains are preserved in comparison to their work. With the same entanglement picture we can compare our results with single-chain slip-spring model in the most straightforward way and form a multi-scale computational framework for polymer rheology.

\section{Method}

\section{Original Single-Chain Slip-Spring Model}

The original single-chain slip-spring model[15] describe the dynamics of a Rouse chain consisting of $\mathrm{N}+1$ beads at position rj connected by $\mathrm{N}$ springs. The Rouse chains are constrained by $\mathrm{Z}=\mathrm{N} / \mathrm{Ne}$ evenly distributed slip-springs representing the topological constraints by surrounding chains, where Ne means the average number of Kuhn segments between slip-links. One end of each slipspring defined as a fixed anchoring point at position aj and the other end attached to the Rouse chain at positon sj with a slip ring. The slip-springs can continuously slid from one monomer to another, which imitate the reptation-like motion of the probe chain. Thus the ring's position is $\mathbf{s}_{\mathbf{j}}=\mathbf{r}_{\operatorname{trunc}\left(x_{j}\right)}+\left(x_{j}-\operatorname{trunc}\left(x_{j}\right)\right)\left(\mathbf{r}_{\operatorname{trunc}\left(x_{j}\right)+1}-\mathbf{r}_{\operatorname{trunc}\left(x_{j}\right)}\right)$,

where $\mathrm{xj}$ is the curvilinear abscissa of $\mathrm{j}$-th slip-spring and trunc(x) is the closest integer to $\mathrm{x}$ less than or equal to $\mathrm{x}$. The total potential energy of the chain is :

$$
U=\frac{3 k_{B} T}{2 b^{2}} \sum_{i=0}^{N-1}\left(\mathbf{r}_{i+1}-\mathbf{r}_{i}\right)^{2}+\frac{3 k_{B} T}{2 N_{\mathrm{S}} b^{2}} \sum_{j=1}^{Z}\left(\mathbf{a}_{j}-\mathbf{s}_{j}\right)^{2},
$$

where $\frac{3 k_{B} T}{2 N_{\mathrm{s}} b^{2}}$ is the slip spring stiffness and Ns is a number of monomers in slip-spring, $\mathrm{b}$ is the Kuhn step. With this total potential, the motion of the chain and the slip-springs obeys the following stochastic equation:

$$
\begin{aligned}
\xi \frac{d \mathbf{r}_{i}}{d t}= & \frac{3 k_{B} T}{b^{2}}\left(\mathbf{r}_{i+1}-2 \mathbf{r}_{i}+\mathbf{r}_{i-1}\right)+\mathbf{f}_{i}(t) \\
& +\frac{3 k_{B} T}{N_{\mathrm{S}} b^{2}} \sum_{j: \operatorname{trunn}\left(x_{j}\right)=i}\left(1-\left(x_{j}-\operatorname{trunc}\left(x_{j}\right)\right)\right)\left(\mathbf{a}_{j}-\mathbf{s}_{j}\right), \\
& +\frac{3 k_{B} T}{N_{\mathrm{S}} b^{2}} \sum_{j: \operatorname{trun}\left(x_{j}\right)=i-1}\left(x_{j}-\operatorname{trunc}\left(x_{j}\right)\right)\left(\mathbf{a}_{j}-\mathbf{s}_{j}\right) \\
\xi_{s} \frac{d x_{j}}{d t} & =\frac{3 k_{B} T}{N_{\mathrm{S}} b^{2}}\left(\mathbf{r}_{\operatorname{trunc}\left(x_{j}\right)+1}-\mathbf{r}_{\text {trunc }\left(x_{j}\right)}\right)\left(\mathbf{a}_{j}-\mathbf{s}_{j}\right)+g_{j}(t),
\end{aligned}
$$

where $\xi, \xi_{s}$ is the monomer and slip-spring friction coefficient respectively, $\mathbf{f}_{i}(t)$ and ${ }^{g_{j}(t)}$ is Gaussian white noise with zero mean, variance $\left\langle f_{i}(t) f_{j}\left(t^{\prime}\right)\right\rangle=2 k_{B} T \xi \mathbf{I} \delta\left(t-t^{\prime}\right) \delta_{i j} \quad$ and $\left\langle g_{i}(t) g_{j}\left(t^{\prime}\right)\right\rangle=2 k_{B} T \xi_{s} \mathbf{I} \delta\left(t-t^{\prime}\right) \delta_{i j}$ respectively. 
When one slip-spring slid off the chain, the slip-spring and the corresponding slip-spring will be destroyed and instantaneously two new slip-springs will appear in the system with one at the end of randomly chosen chain. This process represents "constraint release".

\section{Excluded Volume Interactions}

To achieve a multichain version of Likhtman's model for nonhomogeneous polymer melt, we should introduce excluded volume interactions between segments in the slip-spring model. Biondo et al. adopt a mean field version of the excluded volume Hamiltonian discretized on a lattice and the interaction force acting on bead i expressed as below[18]:

$$
\mathbf{F}_{\text {hom }}=-\frac{\kappa_{0} \delta^{3}}{\rho_{0}} \sum_{\mathbf{c}_{i}}\left[\left(\rho\left(\mathbf{c}_{i}\right)-\rho_{0}\right) \nabla_{\mathbf{r}_{i}}\left(\rho\left(\mathbf{c}_{i}\right)\right)\right]
$$

where $\kappa_{0}=-\frac{\rho_{0}}{k_{B} T} \frac{1}{V}\left(\frac{\partial V}{\partial P}\right)_{T}$ is the dimensionless bulk modulus, $\rho_{0}$ is the mean density, $\delta$ is the length of elementary cell of partitioned cubic lattice and $\mathbf{c}_{i}$ means the lattice node set nearest neighbors of the bead i. $\rho\left(\mathbf{c}_{i}\right)$ is given in eqs (23) in [18] and the detailed implementation we refer to that paper. Here we use $\mathrm{P}=4$ which can recover precisely the relaxation modulus without excluded volume as shown in [18].

\section{Fluctuations in the Number of Entanglements}

For the slip links renewal rules, the number of slip-springs in Likhtman's model is constant. To achieve fluctuation entangments, Uneyama developed a GPU version of this model using grand canonical type ensemble for slip-springs which is orignially introduced by Schieber. The grand potential of the system is $J=U-v Z$, where $v$ is effective chemical potential. The motion equation of beads in the chain is still eq (2). The difference is, using Monte Carlo (MC) method of grand canoniacal ensemble, the slip-springs perform discrete jumps along the polymer beads and $x_{j}$ is assumed as an integer value. We will use this strategy in completing the multchain version model and the detail of the multichain will be implemented with following steps:

1. Sampling the model: generate polymer conformation by Gaussian distribution, sample $\mathrm{Z}$ from Poisson distribution, generate $\mathrm{Z}$ slip-springs by uniform distribution and each anchoring point from the Gaussian distribution. The concrete form of the distributions above refer to [16].

2. Polymer configurations are updated via dynamic simulations:

$$
\begin{aligned}
\xi \mathbf{r}_{i}(t+\Delta t) & =\left[\frac{3 k_{B} T}{b^{2}}\left(\mathbf{r}_{i+1}-2 \mathbf{r}_{i}+\mathbf{r}_{i-1}\right)+\mathbf{f}_{i}(t)\right. \\
\left.\mathbf{F}_{\text {hom }}^{i}+\boldsymbol{\kappa}(t) \cdot \mathbf{r}_{i}+\frac{3 k_{B} T}{N_{\mathrm{S}} b^{2}} \sum_{j: x_{j}=i}\left(\mathbf{a}_{j}-\mathbf{r}_{i}\right)\right] &
\end{aligned}
$$

$\mathbf{F}_{\text {hom }}^{i}$ is computed by eq (4). ${ }^{\boldsymbol{\kappa}(t)}$ is the velocity gradient tensor for the flow condition. In the absence of the velocity gradient, the eq(5) is equivalent to eq(1). And at flow condition, the anchor points change their positions by $\mathbf{a}_{j}(t+\Delta t)=\boldsymbol{\kappa}(t) \cdot \mathbf{a}_{j} \Delta t$.

3. For the slip-spring's position $\left\{x_{j}\right\}$, the MC method of grand canoniacal ensemble is used.For each slip-spring of each chain, move $\mathrm{j}$-th slip-spring from one polymer bead ${ }^{x_{j}}$ to a neighboring one $x_{j} \pm 1$ by transition probabilities $\min \left(\frac{1}{2}, \frac{\Delta t}{\xi_{s}}\left[1-\tanh \left[\frac{3}{4 N_{s}}\left[\left(\mathbf{r}_{x_{j} \pm 1}-\mathbf{a}_{j}\right)^{2}-\left(\mathbf{r}_{x_{j}}-\mathbf{a}_{j}\right)^{2}\right]\right]\right]\right)$,

the probability to propose the move $x_{j} \rightarrow x_{j}+1$ or $x_{j} \rightarrow x_{j}-1$ is equal to $\frac{1}{2}$. For each chain, if one of the rings reaches the chain end, then an existing slip-spring pair will be destroyed with 
probability $\min \left(\frac{1}{2}, \frac{\Delta t}{\xi_{s}}\left(\delta_{x_{j}, 0}+\delta_{x_{j}, N}\right)\right)$. Then create a new slip-spring pair, with one at a randomly selected chain end with probability $\min \left(\frac{1}{2}, \frac{\Delta t}{\xi_{s}} \frac{1}{N_{e}}\right)$ and place the corresponding anchor point according Gaussian distribution in eq(1). As shown in [16], this form of dynamics satisfy the detailed balance condition. The multi-chain slip-spring model is shown schematically in Figure 1.

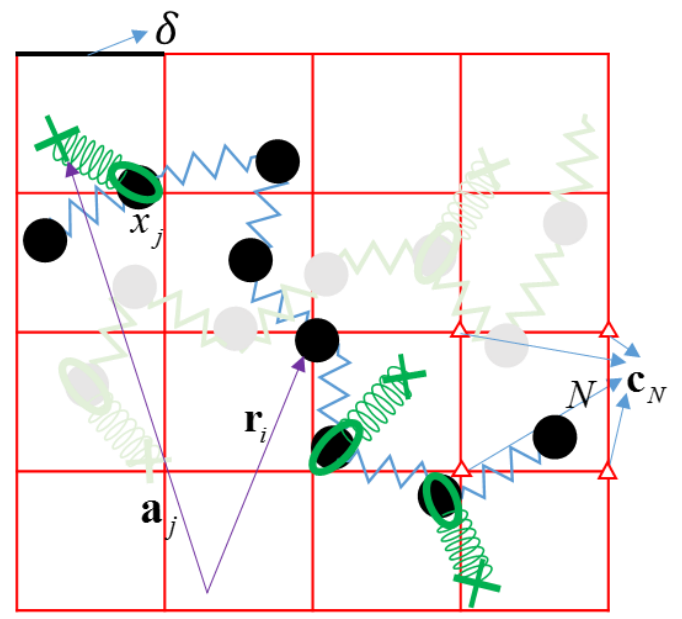

Figure 1.Multi-chain slip-spring model. Two chains are shown in the figure: one light and one dark with a bead position ri, anchor position aj, curvilinear abscissa $x j$ of $j$-th slip-spring. The length of elementary cell of partitioned cubic lattice is $\delta$. The lattice node set ${ }^{\mathbf{c}_{N}}$ containing nearest neighbors of the bead $\mathrm{N}$ are shown with four hollow triangles when $\mathrm{P}=2$.

The parameters used in this model are nondimensionalized. We set the monomer size $b=1$, temperature $^{k_{B} T=1}$, friction coefficient of the entropic springs $\xi=1$, the friction coefficient of the slip-springs $\xi_{S}=0.1$, and characteristic time $\tau^{\tau_{0}=\frac{\xi b^{2}}{k_{B} T}}$.We use $\mathrm{N}=32$ for all the simulations. According to reference [15], $\mathrm{Ne}=4, \mathrm{Z}=8$ are used if not otherwise specified. For the excluded volume interaction, following reference [18] $\rho_{0}=5.98, \kappa_{0}=50 / N=1.5625$ are used. For the dynamics, $\Delta t$ during each step is set as 0.01 .

\section{Results}

First, we check the dynamics of homogeneous polymer melt model with and without excluded volume interaction. The density distribution of the model with exluded volume interaction satisfies Gaussian distribution (as shown in Figure 2), which verifies the correctness of the program involved with the excluded volume interaction partly. The time evolution of shear relaxation modulus of this model and Uneyama's model are shown in Figure(3). The relaxation function $G(t)$ is calculated using the off-diagonal stress-stress correlation function according to the fluctuation-dissipation theorem. Considering the contribution of slip-spring to the stress, the concrete expression of $G(t)$ is [20]:

$$
G(t)=\frac{V}{k_{B} T} \frac{1}{3}\left\langle\sum_{\alpha=1}^{2} \sum_{\beta>\alpha}^{3} \sigma_{\alpha \beta}^{R}(t)\left(\sigma_{\alpha \beta}^{R}(0)+\sigma_{\alpha \beta}^{S L}(0)\right)\right\rangle
$$

$$
\text { Here } \sigma_{\alpha \beta}^{R}=-\frac{3 k_{B} T}{V b^{2}} \sum_{i=1}^{N}\left\langle\left(r_{\alpha, i}-r_{\alpha, i-1}\right)\left(r_{\beta, i}-r_{\beta, i-1}\right)\right\rangle \text { and } \sigma_{\alpha \beta}^{S L}=-\frac{3 k_{B} T}{N_{S} V b^{2}} \sum_{j=1}^{Z}\left\langle\left(r_{\alpha, x_{j}}-a_{\alpha, j}\right)\left(r_{\beta, x_{j}}-a_{\beta, j}\right)\right\rangle \text { reperesent the }
$$
contributions arising from Rouse and slip-spring potential, respectively. We use ${ }^{r_{\alpha, i}}$ as the $\alpha$ coordinate of bead i. G(t) calculated by both model agrees well with same plateau modulus and 
same longest relaxation time. The plateau modulus $G_{N}^{(0)}$ is about $0.1 \rho_{0} k_{B} T$, which is quantitatively consistent with original slip-spring model[15]. As also declared in [18], for the $\mathrm{P}=4$ scheme, the relaxation modulus could be in accordance with the results without excluded volume. Hence the method can reflect the excluded volume interaction correctly without perturbing the dynamics of the chains. For the consideration of excluded volume interaction is based on the work of the group of Barrat [18] and we successfully use it in a multi-chain version model with fluctuating entanglements, thus nanocomposite melt with fillers with grafted chains would be simulated with this model just as their another paper[21]. As pointed out before, this multichain slip-spring model is easily extented from the single-chain slip-spring program and the comparison between both is straightforward.

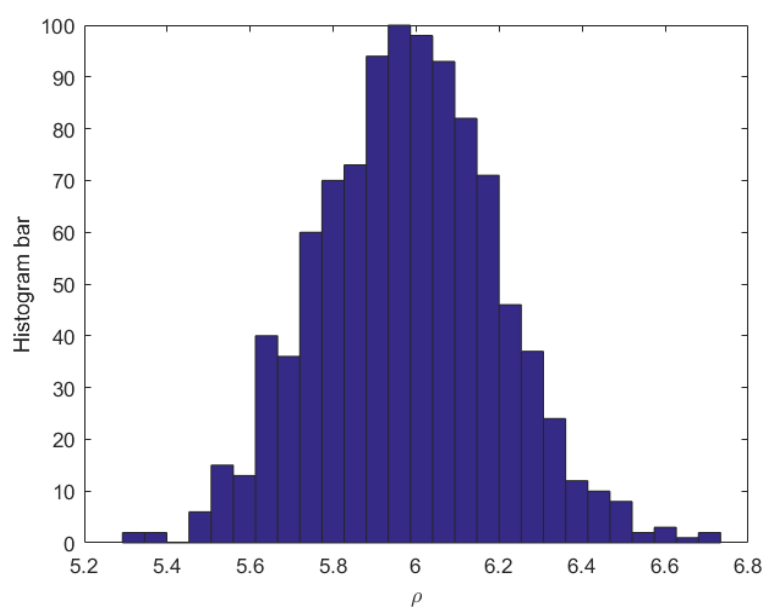

Figure 2. Monomer density distribution satisfies Gaussian distribution with a mean value of $\rho_{0}=5.98$.

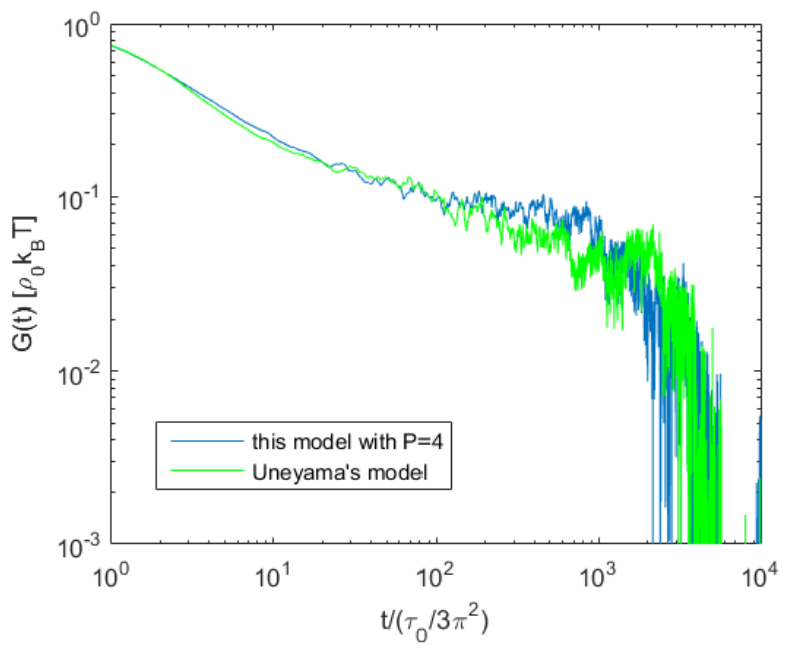

Figure 3. Stress relaxation modulus against time for Uneyama's model of an ensemble of 1000 chains and for multi-chain slip-spring model in this work with 1000 chains in a box.

Next we monitor the number of entanglements to verify the Monte Carlo (MC) method of grand canoniacal ensemble used in this paper. The ensemble contains 1000 chains with $N=32$. We first set the number of entanglements of each chain to 8. After 5000 time steps, the distribution of $\mathrm{Z}$ is shown in Figure 4. As already predicted by Schieber[17], Z follows a Poisson distribution and the Poisson distribution for $\mathrm{Z}=8$ is also shown for comparison. The simulation results agree with the theoretical prediction. The fluctuation of entanglements during the simulation is also shown in the inset. The dynamics satisfies the detailed balance condition and $\mathrm{Z}$ fluctuates around $\langle\mathrm{Z}\rangle=8$. The 
achievement of fluctuation in entanglement number is not trivial and the shear-induced "disentanglement" would be revealed by this complementation, which we will show later.

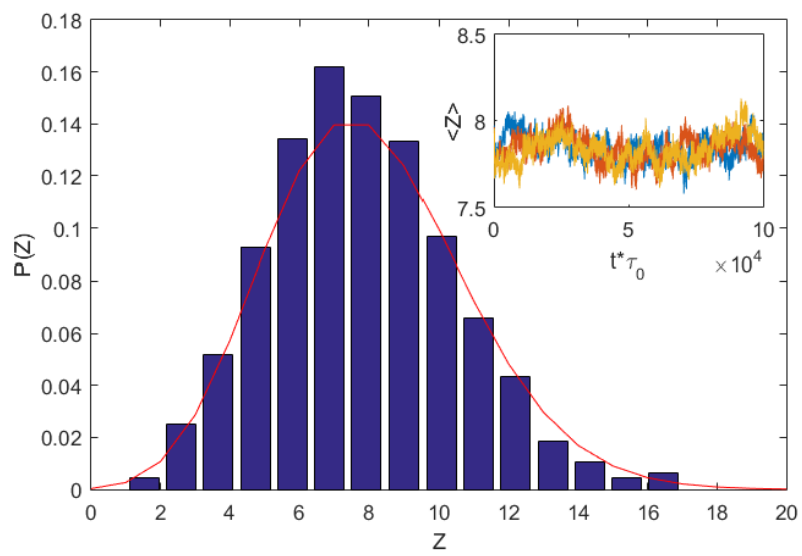

Figure 4. Z follows Poisson distribution with mean N/Ne. Poisson distribution for $Z=8$ is also shown for comparison (red line). The fluctuation of entanglement number during simulation are shown in the inset.

The nonlinear behaviour are also checked using this model. A steady shear flow with a constant shear rate $\dot{\gamma}\left(\dot{\gamma} \tau_{0}=0,0.0025,0.005,0.01,0.025,0.05,0.1\right)$ is imposed on the box. Lee-Edwards periodic boundary conditions are applied to all the beads. Figure 5 shows the shear stress $\sigma_{12}$ vs time with different shear rates. We consider both the Rouse contribution and slip-spring contribution to the instantaneous shear stress here. For larger shear rates, the overshoot behavior can be caught by the model clearly. For lower shear rates, the shear stress became monotonous without overshoot behaivor.

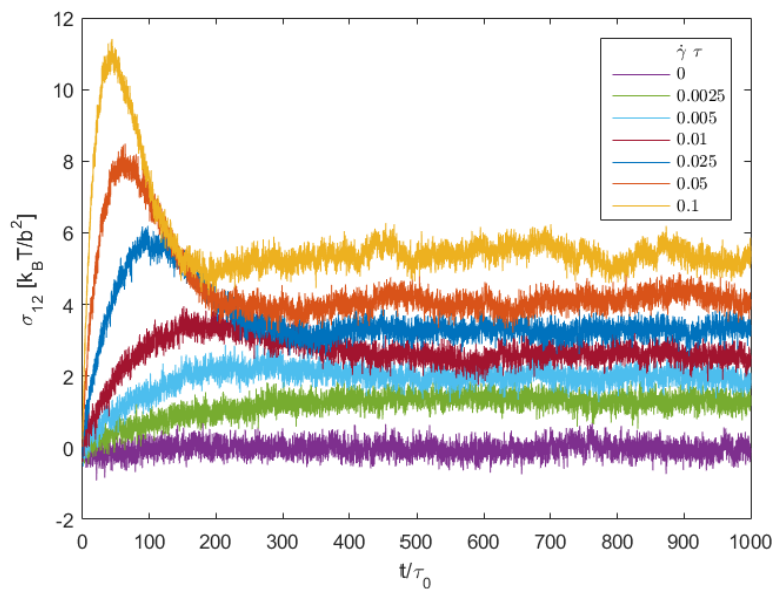

Figure 5. Shear stress vs time with different shear rates.

The average entanglement number $\mathrm{Z}$ was monitored during the flow of different shear rate. When applied a steady shear flow, $\langle Z\rangle$ decrease with time. Figure 6 shows $Z$ vs time for different shear rates, in which the "disentanglement" effect is significant at larger shear rate. This is because at high shear rate, slip-spring were stretched strongly and easily accumulated at the ends of the chain, thus be easily destructed. This was consistent with the work of $[9,19]$ and could not be caught by the model of Biondo[18]. 


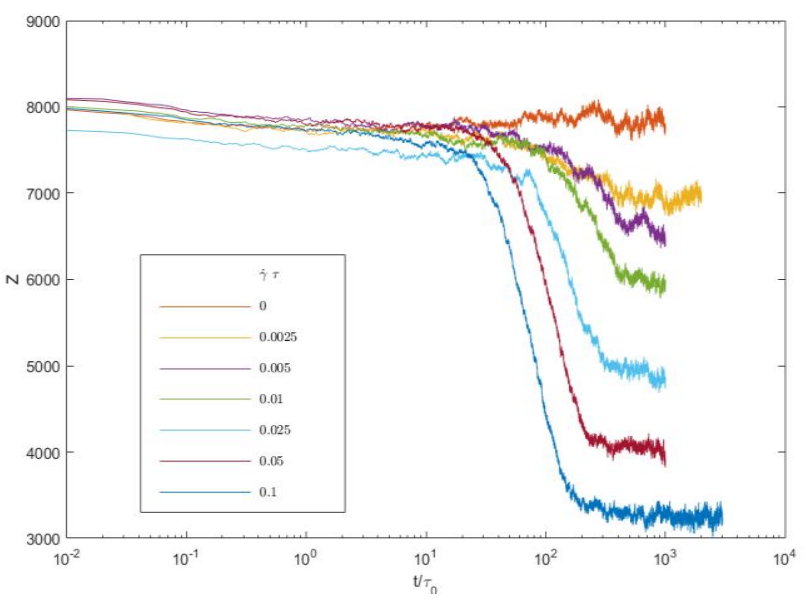

Figure 6. The average entanglement number $\mathrm{Z}$ vs time with different shear rates.

\section{Conclusion}

In this work, we have proposed a multi-chain slip-spring model, which is based on Likhtman's single-chain slip-spring model[15]. The excluded volume interactions is achieved in a mean-field manner. With this introduction, the computed homogeneous melt density satisfy Gaussian distribution and the relaxation modulus agrees well with single-chain slip-spring model, which verifies the dynamically correctness of excluded volume interactions without perturbing the dynamics of the chains. This implementation makes the model go beyond single-chain theory and can be used for dynamics of inhomogeneous system. In contrast to the original Likhtman's model[15] and the extended version used for nanocomposites of Biondo's[18], the number of slipsprings in this model is allowed to fluctuate using MC method of grand-canonical ensemble. The entanglement number $\mathrm{Z}$ follows Possion distribution under equilibrium states. This implementation can also predict the decrease of the number of slip-spring in high shear rates flow.

This model can predict non-homogeneous polymer melts and is easily extended to various chain architectures, such as nanocomposite with fillers with grafted chains using similar method of Masnada[21]. Due to the relationship between this model and Likhtman's single-chain slip-spring model[15], a systematic multi-scale strategy for polymer dynamics is straightforward. And these will be the future work.

\section{Acknowledgement}

The authors are grateful to Prof. Jean-Louis Barrat and Prof. Abelardo Ramírez-Hernández for their helpful discussion.

\section{References}

1. M. Doi S. Edwards, Journal of the Chemical Society, Faraday Transactions 2: Molecular and Chemical Physics. 74, 1789-1801.(1978)

2. M. Doi S. Edwards, Journal of the Chemical Society, Faraday Transactions 2: Molecular and Chemical Physics. 74, 1818-1832.(1978)

3. M. Doi S. Edwards, Journal of the Chemical Society, Faraday Transactions 2: Molecular and Chemical Physics. 74, 1802-1817.(1978)

4. M. Doi S. Edwards, Journal of the Chemical Society, Faraday Transactions 2: Molecular and Chemical Physics. 75, 38-54.(1979)

5. J. Des Cloizeaux, EPL (Europhysics Letters). 5, 437.(1988)

6. R. Ketzmerick H. Öttinger, Continuum Mechanics and Thermodynamics. 1, 113-124.(1989) 
7. G. Marrucci, Journal of non-newtonian fluid mechanics. 62, 279-289.(1996)

8. Y. Masubuchi, et al., The Journal of Chemical Physics. 115, 4387.(2001)

9. Y. Masubuchi, et al., J Chem Phys. 128, 154901.(2008)

10. A. Ramírez-Hernández, et al., Macromolecules. 46, 6287-6299.(2013)

11. A. Ramírez-Hernández, M. Müller, J. J. de Pablo, Soft Matter. 9, 2030.(2013)

12. J. D. Schieber, J. Neergaard, S. Gupta, Journal of Rheology. 47, 213.(2003)

13. F. Xu, M. M. Denn, J. D. Schieber, Journal of Rheology. 50, 477.(2006)

14. R. N. Khaliullin J. D. Schieber, Macromolecules. 42, 7504-7517.(2009)

15. A. E. Likhtman, Macromolecules. 38, 6128.(2005)

16. T. Uneyama, Nihon Reoroji Gakkaishi. 39, 135-152.(2011)

17. J. D. Schieber, The Journal of Chemical Physics. 118, 5162.(2003)

18. D. Del Biondo, et al., J Chem Phys. 138, 194902.(2013)

19. A. Ramirez-Hernandez, et al., J Chem Phys. 143, 243147.(2015)

20. J. Ramirez, S. K. Sukumaran, A. E. Likhtman, J Chem Phys. 126, 244904.(2007)

21. E. Masnada, et al., Soft Matter. 9, 10532.(2013) 